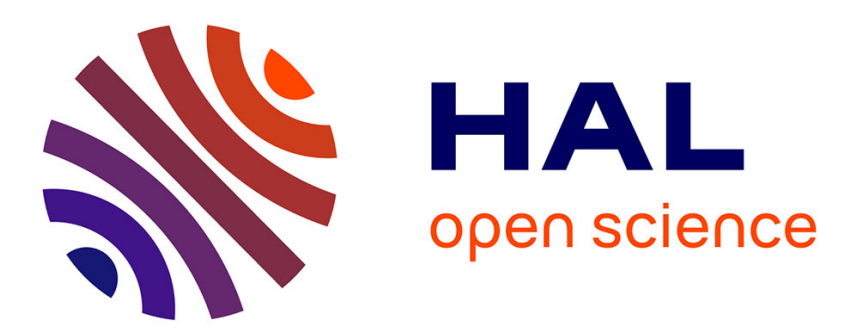

\title{
Cinétique de croissance et propriétés électriques des couches de silice obtenues sous irradiation infrarouge \\ Gérard Sarrabayrouse, A. Guedj
}

\section{To cite this version:}

Gérard Sarrabayrouse, A. Guedj. Cinétique de croissance et propriétés électriques des couches de silice obtenues sous irradiation infrarouge. Revue de Physique Appliquée, 1988, 23 (6), pp.1135-1138. 10.1051/rphysap:019880023060113500 . jpa-00245916

\section{HAL Id: jpa-00245916 https://hal.science/jpa-00245916}

Submitted on 1 Jan 1988

HAL is a multi-disciplinary open access archive for the deposit and dissemination of scientific research documents, whether they are published or not. The documents may come from teaching and research institutions in France or abroad, or from public or private research centers.
L'archive ouverte pluridisciplinaire HAL, est destinée au dépôt et à la diffusion de documents scientifiques de niveau recherche, publiés ou non, émanant des établissements d'enseignement et de recherche français ou étrangers, des laboratoires publics ou privés. 


\title{
Cinétique de croissance et propriétés électriques des couches de silice obtenues sous irradiation infrarouge
}

\author{
G. Sarrabayrouse et A. Guedj \\ Laboratoire d'Automatique et d'Analyse des Systèmes du C.N.R.S., 7 avenue du Colonel Roche, 31077 \\ Toulouse Cedex, France
}

(Reçu le 7 janvier 1988, accepté le 29 février 1988)

\begin{abstract}
Résumé. - La cinétique de croissance ainsi que les propriétés électriques des couches de silice obtenues sous illumination IR ont été étudiées. Il apparaît que la vitesse de croissance est identique à celle d'une couche obtenue par oxydation thermique dans la gamme de température $950-1050^{\circ} \mathrm{C}$. De plus, leurs propriétés électriques se comparent favorablement à celles de leurs homologues thermiques.
\end{abstract}

Abstract. - The growth kinetics and the electrical properties of silica layers obtained under infrared illumination have been studied. The growth rate is identical to that of thermal oxide layers in the range $950^{\circ} \mathrm{C}$ $1050^{\circ} \mathrm{C}$. Furthermore their electrical properties compare favorably with those of thermal oxide layers.

\section{Introduction.}

Dans le cadre du développement de nouvelles méthodes de préparation des couches minces en technologie silicium, l'utilisation de sources lumineuses laser ou incohérentes reçoit un intérêt de plus en plus important. En ce qui concerne la croissance de couches de silice, de nombreuses études ont mis en évidence les possibilités offertes par une irradiation infrarouge, visible ou ultraviolette du silicium en milieu oxygéné $[1,2]$.

Dans ce cadre, l'utilisation d'un laser continu au $\mathrm{CO}_{2}$ a fait l'objet de plusieurs travaux particulièrement par Boyd et al. qui ont mis en évidence un meilleur ordre structural des couches de silice ainsi obtenues comparées à celles issues d'une oxydation thermique classique [3]. Ce résultat prend toute son importance du fait de la très faible épaisseur des couches de silice utilisées actuellement $(<100 \AA)$ dans certains dispositifs mettant en exergue le rôle de la zone désordonnée et contrainte de l'interface $\mathrm{SiO}_{2}-\mathrm{Si}$.

Cependant, peu de résultats concernent les propriétés électriques des couches de silice ainsi obtenues en particulier au niveau du champ de claquage [1], paramètre d'autant plus important que la couche est mince. Par ailleurs, le claquage dépend fortement des conditions de mesure (surface de l'échantillon, condition de polarisation, critère de détection du claquage) [4], et des conditions de fabrication de la couche (ambiance d'oxydation, présence d'impuretés, nature de l'électrode) [5-7]. De ce fait, une évaluation des potentialités des couches obtenues $\left(\mathrm{L}-\mathrm{SiO}_{2}\right)$ par comparaison avec des couches standard $\left(\mathrm{T}-\mathrm{SiO}_{2}\right)$ ne peut être menée sur la base de travaux effectués dans des conditions et par des auteurs différents.

En ce qui concerne la croissance des couches, aucun résultat ne figure dans la littérature sur la mesure directe de l'influence de la température sur la cinétique d'oxydation. Ceci est dû à la difficulté de mesurer la température de surface de l'échantillon durant l'oxydation sous irradiation [3].

Dans ce contexte, cet article présente les résultats relatifs à la cinétique d'oxydation et aux propriétés électriques des couches obtenues. L'influence de la température sur la vitesse de croissance est analysée, ce qui permet d'étayer les éléments avancés dans la littérature quant au mécanisme d'oxydation.

Les propriétés électriques quant à elles sont comparées à leurs homologues obtenus sur des couches de silice élaborées par voie thermique dans la même atmosphère et la même enceinte.

\section{Conditions expérimentales.}

Les couches de silice $\mathrm{L}_{-} \mathrm{SiO}_{2}$ sont obtenues sous atmosphère d'oxygène sec dans une enceinte en 
acier inoxydable munie d'une fenêtre en $\mathrm{ZnSe}$, d'une fenêtre en pyrex permettant la mesure de la température et d'un support assurant le préchauffage de l'échantillon par effet Joule. Le substrat est irradié par un laser $\mathrm{CO}_{2}$ continu $(\lambda=10.6 \mu \mathrm{m}) \mathrm{du}$ type CILAS 170 ayant une puissance de $10 \mathrm{~W}$ au travers d'une lentille en ZnSe permettant d'atteindre sur l'échantillon une densité de puissance variable de $100 \mathrm{~W} \mathrm{~cm}^{-2}$ à $500 \mathrm{~W} \mathrm{~cm}^{-2}$.

Le préchauffage est nécessaire afin d'assurer une absorption suffisante du faisceau laser [8] et de réduire le gradient thermique à la surface du substrat.

Les couches $\mathrm{T}-\mathrm{SiO}_{2}$ sỏnt obtenues dans la même enceinte par oxydation thermique, le chauffage par effet Joule permettant d'atteindre des températures supérieures à $1000^{\circ} \mathrm{C}$. Ces éléments sont montés sur un banc optique associés à un calorimètre Ophir $390 \mathrm{~A} / \mathrm{W}$, un pyromètre Chauvin et Arnoux monochromatique à $\lambda=0,65 \mu \mathrm{m}$, un pyromètre infrarouge Gulton Infratherm travaillant dans la bande $\lambda=1-3 \mu \mathrm{m}$ et une caméra infrarouge Hughes TVS 4100 travaillant dans la bande $2-6 \mu \mathrm{m}$.

Le calorimètre a été utilisé pour déterminer le profil énergétique du faisceau et pour contrôler (associé à une lame semi-réfléchissante) la stabilité de la puissance totale du faisceau à l'entrée de l'enceinte durant l'oxydation.

Le pyromètre monochromatique a été utilisé pour la mesure de la température de surface de l'échantillon durant l'oxydation. Il a été étalonné par relevé de la température de luminance d'échantillons de silicium oxydés dans l'enceinte par oxydation thermique pendant 10 à 120 minutes à différentes températures de luminance. La température vraie de ces échantillons dans la gamme $900-1000^{\circ} \mathrm{C}$ a été obtenue par comparaison des courbes de cinétique avec les résultats de simulation d'oxydation sous oxygène $\mathrm{sec}$ issus du programme SUPREM III. De plus, ces résultats et ceux issus d'une mesure de la température d'un four assimilé à un corps noir [9] permettent d'obtenir l'émissivité monochromatique du silicium à $\lambda=0,65 \mu \mathrm{m}$ et dans les conditions de mesure. La valeur 0,7-0,72 obtenue est en accord avec les résultats figurant dans la littérature $(0,58-0,75)$ [10] concernant le silicium. Cependant, la valeur obtenue est légèrement supérieure aux valeurs généralement rencontrées pour une surface de silicium polie, la mesure étant faite sous vide [10]. Ceci peut être dû à la croissance de la couche d'oxyde comme cela a été mis en évidence dans le cas des métaux [11].

Le pyromètre infrarouge a été utilisé pour s'assurer de la stabilité de la température de l'échantillon durant l'oxydation et pour évaluer la température de préchauffage $T_{0}$.

Cette dernière mesure ne donne qu'une valeur très approchée de la température du substrat endessous de $700^{\circ} \mathrm{C}$. En effet, l'émissivité du silicium dans la bande 1-3 $\mu \mathrm{m}$ pour un dopage de l'ordre de $10^{15} \mathrm{~cm}^{-3}$ et pour de telles températures varie de 0,4 à 0,7 [12] en fonction de la longueur d'onde, ce qui n'autorise pas une mesure précise de la température. Cependant, en ce qui concerne les conditions de préchauffage, leur reproductibilité a été contrôlée par l'intermédiaire de la valeur du courant de préchauffage $I_{0}$ et par l'uniformité de l'image thermographique donnée par la caméra IR.

Les échantillons testés sont des capacités MOS réalisées sur un substrat de silicium épitaxié de type $\mathrm{N} / \mathrm{N}^{+}$d'orientation $\langle 100\rangle$ de résistivité $5 \Omega \mathrm{cm} / 10^{-3} \Omega \mathrm{cm}$. Après un nettoyage préoxydation standard [13], le substrat est introduit dans l'enceinte où il est oxydé par l'une ou l'autre des deux méthodes. Après oxydation $500 \AA \AA$ de chrome et $2000 \AA$ d'or sont successivement déposés et gravés afin de définir des motifs de $10^{-4} \mathrm{~cm}^{2}$ de surface avec une densité de 2500 dispositifs par $\mathrm{cm}^{2}$. La face arrière du substrat est ensuite désoxydée puis métallisée à l'or.

Les conditions d'obtention des couches de silice sont décrites dans le tableau suivant dans lequel les séries marquées $i L$ correspondent à une oxydation laser avec une température de préchauffage $T_{0}$, une densité de puissance au centre du faisceau laser incident égale à $P_{\max }$ et une température sous irradiation égale à $T$. Les séries marquées $i T$ correspondent à une oxydation thermique à la température $T$.

Tableau I. - Condition de croissance des couches.

\begin{tabular}{|c|c|c|r|}
\hline Série & $P_{\max }\left(\mathrm{W} \mathrm{cm}^{-2}\right)$ & $T_{0}\left({ }^{\circ} \mathrm{C}\right)$ & $T\left({ }^{\circ} \mathrm{C}\right)$ \\
\hline $1 \mathrm{~L}$ & 150 & 600 & 950 \\
2L & 500 & 300 & 950 \\
$4 \mathrm{~L}$ & 150 & 700 & 1000 \\
$5 \mathrm{~L}$ & 500 & 450 & 1000 \\
$7 \mathrm{~L}$ & 500 & 550 & 1050 \\
3T & - & - & 950 \\
$6 \mathrm{~T}$ & - & - & 1000 \\
\hline
\end{tabular}

La couche de silice ainsi obtenue a une épaisseur non uniforme dont le profil épouse celui de la répartition de puissance du faisceau incident. Les seuls dispositifs pris en compte pour les caractérisations électriques se trouvent dans la zone centrée sur le faisceau et correspondent à une épaisseur de silice supérieure à 0,9 fois l'épaisseur maximale.

Les dispositifs sont caractérisés électriquement sur la base des courbes capacité tension $C$ - $V$ obtenues à $1 \mathrm{kHz}$ et $1 \mathrm{MHz}$ et des courbes courant-tension $I-V$ obtenues lors d'une polarisation par une rampe de tension.

Suivant une procédure maintenant classique [14], l'épaisseur de la couche de silice et la densité d'états 
de surface à l'interface $\mathrm{SiO}_{2}$-Si sont extraites des courbes $C-V$. Le mécanisme de conduction, la hauteur de barrière métal-isolant et le champ de claquage de la couche sont extraits de la caractéristique $I-V$.

\section{Résultats expérimentaux et discussions.}

3.1 CinétiQue DE CROISSANCE. - La cinétique de croissance des couches obtenues dans les conditions précisées dans le tableau I, de même que les résultats de simulation par le programme SUPREM III utilisant le modèle d'oxydation sèche standard sont représentés sur la figure 1 . Celle-ci montre que l'accord entre les trois types de résultat est satisfaisant.

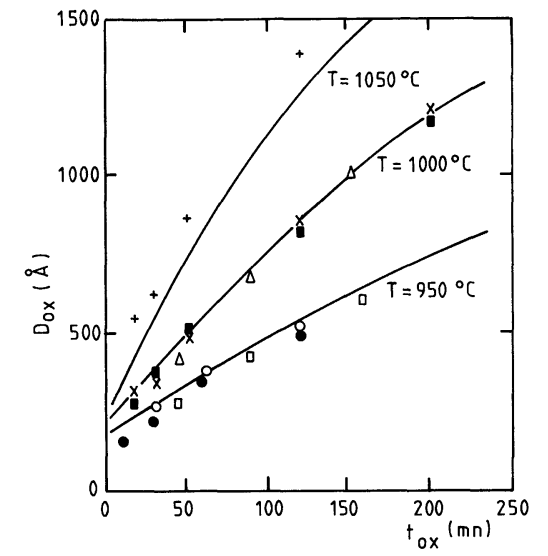

Fig. 1. - Cinétique de croissance des couches $\mathrm{L}_{-} \mathrm{SiO}_{2}$ et $\mathrm{T}_{-\mathrm{SiO}_{2}}((\bullet) 1 \mathrm{~L} ;(0) 2 \mathrm{~L},(\times) 4 \mathrm{~L},(\Delta) 5 \mathrm{~L},(+) 7 \mathrm{~L},(\square) 3 \mathrm{~T}$, (ש) $6 \mathrm{~T},(-)$ simulation).

[Growth kinetics of $\mathrm{L}^{-\mathrm{SiO}_{2}}$ and $\mathrm{T}-\mathrm{SiO}_{2}$ layers.]

Les résultats expérimentaux pour $T=1050^{\circ} \mathrm{C}$ montrent un écart par rapport à la simulation de l'ordre de $20^{\circ} \mathrm{C}$. Ceci correspond à une variation d'émissivité de $5 \times 10^{-2}$. Or, l'émissivité du silicium pour $\lambda=0,65 \mu \mathrm{m}$ décroit lorsque la température augmente [10]. Ainsi, il n'apparait pas improbable qu'une surestimation de l'émissivité ait conduit à une sous-estimation de la température. En effet, la valeur 0,71 , valeur moyenne dans la gamme $900-$ $1000^{\circ} \mathrm{C}$ a été utilisée pour la mesure de la température en dehors de cette gamme.

En conséquence, la cinétique de croissance des oxydes obtenus sous-irradiation coïncide avec celle des oxydes thermiques.

De plus, la comparaison entre les séries $1 \mathrm{~L}$ et $2 \mathrm{~L}$ d'une part, $4 \mathrm{~L}$ et $5 \mathrm{~L}$ d'autre part montre que la densité de puissance incidente est sans effet sur la cinétique de croissance dès lors que la température de l'échantillon est constante.

Ces résultats confirment donc les éléments, avancés dans [15] sans que l'influence de la température sur la vitesse de croissance ait été directement mesurée, selon lesquels l'oxydation sous irradiation au laser $\mathrm{CO}_{2}$ est une oxydation thermique.

3.2 Propriétés ÉlectriQues. - Le relevé des caractéristiques courant-tension montrent (voir Fig. 2) que la conduction est du type Fowler-Nordheim quelle que soit l'épaisseur de la couche de silice dans la gamme d'épaisseur 150-1 $000 \AA$ A. Les hauteurs de barrière Métal-Isolant extraites de la pente de ces caractéristiques tracées dans le plan de Fowler (Fig. 2) [14] en supposant la masse effective des électrons égale à $0,5 \mathrm{~m}_{0}$ où $\mathrm{m}_{0}$ est la masse de l'électron libre, se situent entre $2,9 \mathrm{eV}$ et $3,13 \mathrm{eV}$ en bon accord avec les valeurs obtenues dans ce travail sur les oxydes thermiques $(2,9 \mathrm{eV}-3,1 \mathrm{eV})$ ou publiées dans la littérature $(3,1 \mathrm{eV}-3,2 \mathrm{eV})$ [16].

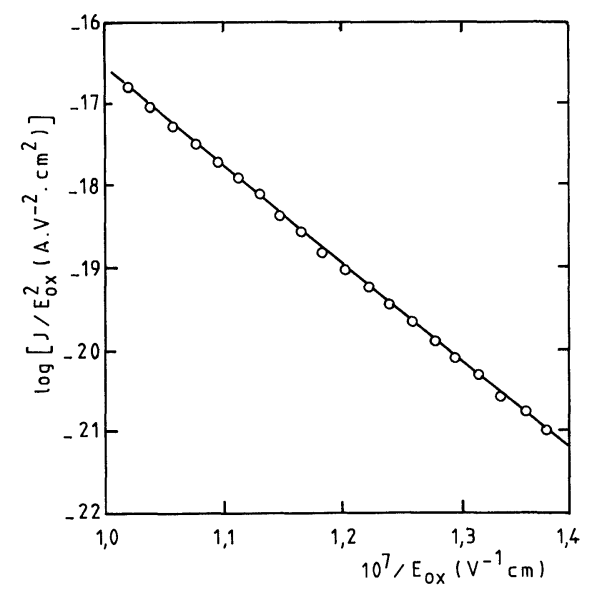

Fig. 2. - Exemple de caractéristique courant-tension tracée dans le plan de Fowler ; $D_{\mathrm{ox}}=150 \AA$; $E_{\mathrm{ox}}$ : champ électrique ; $J$ : courant.

[Example of a current-voltage curve in the Fowler graph ; ( $D_{\mathrm{ox}}=150 \AA ; E_{\mathrm{ox}}$ : Electric Field ; J : current).]

En ce qui concerne le champ de claquage $E_{\mathrm{BD}}$ le caractère localisé de l'oxydation n'a pas permis une étude statistique et seuls 20-30 composants ont été testés pour chaque couche. Cependant à des fins de comparaison et bien que la procédure soit pénalisante, car elle ne prend pas en compte la répartition des champs de claquage, nous avons représenté sur la figure 3 la valeur moyenne de $E_{\mathrm{BD}}$ obtenue sur chaque série. Il apparaît clairement sur cette figure que les couches de silice obtenues sous illumination présentent un champ de claquage supérieur à celui des couches de silice thermiques. De plus, les valeurs maximum enregistrées sont proches des valeurs maximum publiées pour les oxydes thermiques dans la même gamme d'épaisseur [17].

Enfin, en ce qui concerne la densité d'états de surface à l'interface $\mathrm{SiO}_{2} \mathrm{Si}$, la répartition énergéti- 


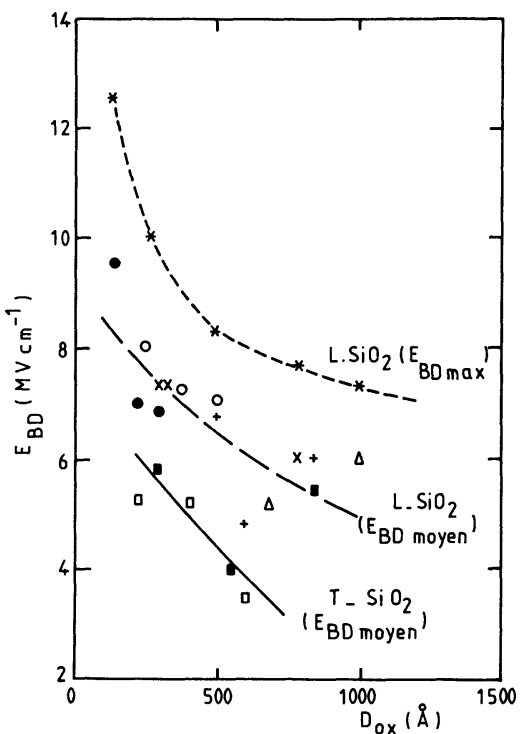

Fig. 3. - Champ de claquage en fonction de l'épaisseur de la couche de silice (mêmes symboles que pour la Fig. 1).

[Breakdown voltage versus silica layer thickness (same symbols as in Fig.1).]

que présente un maximum pour une énergie située à $0,45 \mathrm{eV}$ du fond de la bande de conduction du semiconducteur. La valeur moyenne obtenue en ce point pour les deux types de couche est représentée sur la figure 4 qui montre une densité inférieure dans le cas des oxydes obtenus sous illumination laser.

Ainsi, il apparaît que du point de vue des propriétés électriques, les couches de silice obtenues sous illumination par un laser $\mathrm{CO}_{2}$ se comparent favorablement à leurs homologues thermiques. Ceci est à rapprocher des résultats obtenus par J. W. Boyd et

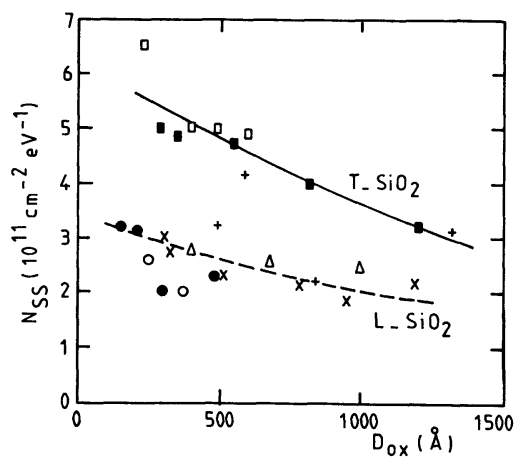

Fig. 4. - Densité d'états de surface en fonction de l'épaisseur de la couche de silice (mêmes symboles que pour la Fig. 1).

[Surface states density versus silica layer thickness (same symbols as in Fig. 1).]

ses collaborateurs [18] et par nous-mêmes sur les échantillons étudiés ici, lors d'une analyse de spectres d'absorption IR dont les résultats montrent un meilleur ordre structural des couches $\mathrm{L}_{-} \mathrm{SiO}_{2}$. Enfin, notons que la densité de la silice des couches $\mathrm{L}-\mathrm{SiO}_{2}$ est supérieure à celles des couches $\mathrm{T}-\mathrm{SiO}_{2}$. En effet, les vitesses d'attaque dans une solution ( $\mathrm{HF}, \mathrm{HNO}_{3}, \mathrm{H}_{2} \mathrm{O} ; 1,5: 1$ : 30) ont respectivement été égales à $120 \AA \mathrm{min}^{-1}$ et $125 \AA \mathrm{min}^{-1}$.

\section{Remerciements.}

Les auteurs tiennent à remercier Madame D. BielleDaspet pour ses conseils et les discussions que nous avons eues sur ce sujet ainsi que Madame F. Rossel et Monsieur G. Lacoste pour leur aide lors de la préparation des échantillons.

\section{Bibliographie}

[1] BoYD, I. W., European Material Research Society Spring Meeting, Ed. G. Bentini, Strasbourg XII (1986) 177.

[2] FogARASSY, E., European Material Research Society Spring Meeting, Strasbourg Ed. V. T. Nguyen et A. G. Cullis IV (1986) 153.

[3] Boyd, I. W. and Wilson, J. I. B., Appl. Lett. 50 (1987) 320

[4] Osburn, C. M. and ORMOND, D. W., J. Electrochem. Soc. 119 (1972) 591.

[5] IrÈnE, E. A., J. Electrochem. Soc. 127 (1980) 396.

[6] Takizawa, R., Nazkanishi, T. and Oshawa, A., Fall Meeting of the Electrochemical Society Honolulu, Hawai 87-2, Abstract $n^{\circ} 713$ (1987) 1017.

[7] Balland, B., Plossu, C., Bardy, S., Thin Solid Films 148 (1987) 149.

[8] Boyd, I. W., Wilson, J. I. B. and West, J. L., Thin Solid Films 83 (1981) L173.
[9] Millet, F., Rev. Gén. Therm. 251 (1982) 879-891.

[10] Allen, F. G., J. Appl. Phys. 28 (1957) 1510.

[11] ANE, J. M., et al. Rev. Int. Hautes Temp. 20 (1983) 187-227.

[12] Sato, T., Jpn. J. Appl. Phys. 6 (1967) 339.

[13] Kern, W. and Puotinen, D. A., RCA Rev. 31 (1970) 187.

[14] Capilla, J. and Sarrabayrouse, G., Revue Phys. Appl. 19 (1984) 343.

[15] BOYD, I. W., Laser processing and Diagnostics Ed. D. Bauerle (Springer, Heidelberg) 1974.

[16] Osburn, C. M. and Weitzman, E. J., J. Electrochem. Soc. 119 (1972) 603.

[17] Osburn, C. M. and ORMOND, D W., J. Electrochem. Soc. 119 (1972) 597.

[18] Boyd, I. W. and Wilson, J. I. B., Appl. Phys. Lett. 41 (1982) 162. 